\title{
ASSOCIATION OF CAROTID INTIMA-MEDIA THICKNESS WITH LEPTIN AND APOLIPOPROTEIN B/APOLIPOPROTEIN A-I RATIO REVEALS IMMINENT PREDICTORS OF SUBCLINICAL ATHEROSCLEROSIS IN PSORIASIS PATIENTS
}

\begin{abstract}
Kumari Asha ${ }^{1}$, Suman B. Sharma ${ }^{1}$, Archana Singal', Amitesh Aggarwal ${ }^{3}$
University College of Medical Sciences and G.T.B. Hospital, Dilshad Garden, University of Delhi, India: Department of Biochemistry ${ }^{1}$, Department of Dermatology ${ }^{2}$ and Department of Medicine ${ }^{3}$

Summary: Psoriasis patients are often susceptible to cardiovascular diseases (CVD), including atherosclerosis. Traditional markers (biochemical and inflammatory) and diagnostic tools could detect occlusive but not subclinical atherosclerosis. Carotid intima-media thickness (CIMT), has recently been recognised as a non invasive diagnostic tool for identification of premature atherosclerosis. Therefore we evaluated 80 psoriasis patients and 80 age sex matched healthy controls for serum leptin levels and apolipoprotein B/apolipoprotein A-I ratio (apoB/apoA-I ratio) in relation with CIMT of carotid artery. Carotid intima-media thickness and carotid plaques were simultaneously measured by carotid sonography. Serum concentration of leptin and apolipoprotein were measured using enzyme-linked immuno sorbent assay (ELISA) and nephelometry respectively. Raised CIMT correlated to age of onset of the disease, serum leptin and apoB/apoA-I ratio in psoriasis patients. Taking into account, values that were above the 75 percentile of the three markers (leptin, apoB/apoA-I ratio and CIMT) the odds ratio was $4.26(2.06-8.80 \mathrm{CI})$. Leptin and apoB/apoA-I ratio showed significant cumulative association with CIMT. Results of predictive analysis supports measurement of CIMT along with estimation of serum leptin and apoB/apoA-I ratio for prediction of premature atherosclerosis in psoriasis patients.
\end{abstract}

Key words: Apolipoprotein; apoB/apoA-I, Leptin; Psoriasis; CIMT; Atherosclerosis

\section{Introduction}

Psoriasis is an immune intervened inflammatory disorder of skin that affects nearly $2-3 \%$ of the total world's population. The disease is caused by unusual differentiation and hyper proliferation of the keratinocytes and can be identified by presence of red, scaly, sharp demarcated plaques mostly over extremities and scalp. Psoriasis is a systemic disease and studies suggest that patients with psoriasis are at increased risk of cardiovascular diseases, including subclinical atherosclerosis (1). The increased incidences of cardiovascular disease in these patients cannot be explained by conventional risk factors (older age, high blood pressure (BP), dyslipidemia, smoking, obesity, diabetes mellitus) alone $(1,2)$. There is an urgent need to study role of other contributory factors common to both psoriasis and cardiovascular disease. Recently, American Association of Clinical Endocrinologists has approved routine measurement of apolipoprotein to assess risk of cardiovascular disease (3). Serum apoA-I (apolipoprotein A-I) is an index of concentration of HDL-C (high-density lipoprotein-cholesterol) in plasma and apoB (apolipoprotein B) is primary apolipoprotein associated with LDL-C (low-density lipoprotein-cholesterol). Thus the balance between the pro-atherogenic apoB and the anti-atherogenic apoA-I can help in the estimation of cardiovascular risk.

Previous studies have shown that both immune cell response and chronic inflammatory cells are involved in accelerating atherosclerotic risk in patients of psoriasis. Immune cell dysfunction in psoriasis produces systemic inflammation. Cytokines involved in inflammation further increases serum leptin, a $16 \mathrm{kDa}$ obese protein, which in turn increases proinflammatory mediators, thus inducing vicious circle of inflammation (4). Thus, we evaluated patients for serum leptin which is known to regulate body weight, metabolism and immunity. Additionally, leptin induces proliferative and anti-apoptotic activities in T cell (5). Since psoriasis is an immune mediated disease, characterized by hyperproliferation of skin cells and infiltration of $\mathrm{T}$ lymphocytes, leptin may provide a link between $T$ cell function and inflammation in psoriasis (6). Clinical studies done so far gave contradictory results, suggesting a multifaceted role of leptin, as an obese protein and/or immunomodulator (decreased immune cell response), in immune-mediated inflammatory conditions in humans. 
CIMT measurement has been validated as a surrogate non invasive diagnostic tool to identify atherosclerosis at sub clinical stage $(7,8)$. Thus, we measured CIMT and evaluated its potential relation with apoB/apoA-I ratio and serum leptin in patients of psoriasis.

\section{Material and Methods}

\section{Human subjects}

The study involved 80 adult patients ( $\geq 18$ years) of either sex, diagnosed to have psoriasis, from Dermatology Outpatient Department, UCMS \& GTB Hospital, Delhi and 80 age, sex and BMI matched healthy volunteers with no known systemic disease. Norms of National Cholesterol Education Program Expert Panel Adult Treatment Panel III for Asian definition was adapted for defining conventional cardiovascular risk factors (smoking, central obesity, dyslipidemia, diabetes, hypertension) in these patients (9).

\section{Inclusion and exclusion criteria}

Patients below the age of 18 years, pregnant and lactating female patients and subjects who had systemic treatment of retinoids in last six months for the management of their psoriasis were excluded from the study. Those who were on lipid-lowering medication were also not included in the study. The control group comprised healthy hospital staff and patients from dermatology outpatients clinics who were not undergoing any systemic therapy nor had been diagnosed with lipid metabolic disorders.

\section{Physical and clinical evaluation of study groups}

Since the present study involves investigations of various conventional and nonconventional risk factors so confounding factors which are known to have an effect on blood lipids value, were recorded. All patients were subjected to detailed personal interview and standardized clinical examination using a detailed questionnaire to obtain information including:

\section{Physical assessment}

Age, sex, Body Mass Index (BMI) [wt (kg)/ height (m) $\left.{ }^{2}\right]$ (10), Waist/ Hip ratio (11).

\section{Clinical evaluation}

Detailed medical and treatment history including the use of lipid-lowering medications, current cigarette smoking status and alcohol consumption. Clinical severity of disease was assessed using Psoriasis Area and Severity Index (PASI) (12).

The study was approved by institutional human ethical committee and a written consent was obtained from all participants of the study.

\section{Sample collection}

Following an overnight fasting, $5 \mathrm{ml}$ of blood sample was drawn from participants using venipuncture, under aseptic conditions. Blood was centrifuged at $2000 \mathrm{~g}$ for 10 minutes and serum sample thus obtained was preserved at $-80{ }^{\circ} \mathrm{C}$ until assayed.

\section{Assay of biochemical parameters}

Fasting and post prandial glucose levels were measured spectrophotometrically at $500 \mathrm{~nm}$ using glucose oxidase-peroxidase method.

Fasting serum lipid profile estimation was done by measuring the level of total serum cholesterol (TC) (enzymatic-calorimetric CHOP-PAP method), serum triglycerides (TG) (enzymatic GPO-PAP method), high density lipoprotein-cholesterol (HDL-C), very low density lipoprotein-cholesterol (VLDL-C) and low density lipoprotein-cholesterol (LDL-C) (calculated using Friedwald and Fredrickson's formula).

Serum concentration of apolipoprotein (apoA-I and apoB) and leptin were measured using kits based on nephelometry (Goldsite Diagnostics Inc, China) and ELISA (BioVendor) respectively as per manufacturer's instructions.

\section{Measurement of Carotid intima media thickness (CIMT)}

CIMT and plaques of both right and left carotid arteries were measured to assess subclinical atherosclerosis and structural changes in the vascular wall using a high resolution B mode ultrasound with a $10 \mathrm{MHZ}$ linear vascular probe (13). All carotid ultrasound measurements were performed by an expert cardiologist. Values above $0.7 \mathrm{~mm}$ were defined as elevated CIMT (14). The mean CIMT was calculated as the arithmetical mean of two measurements of the left and right carotid arteries.

\section{Statistical analysis}

Statistical analysis was carried out using standard statistical software (SPSS software version 20). Quantitative data were expressed as mean \pm standard deviation . Comparison between patients and controls for different parameters was done using t-test. Pearson correlation analysis was used to determine the relation of CIMT with other risk variables. The difference between patients with psoriasis and control group under study has been explained using test of equality of correlations coefficients. We generated receiver operator characteristic (ROC) curve for serum leptin, apoB/ apoA-I ratio and mean CIMT. Specificity, sensitivity and predictive values were calculated using MedCalc statistical software. Association of PASI with CIMT was investigated using adjusted logistic regression model. Multivariate regression analysis was used to study the effect of inde- 
pendent (predictor) variables on one dependant (CIMT) variables. For all statistical tests, $\mathrm{P}<0.05$ was considered significant.

\section{Results}

\section{Clinical characteristics}

A total of 80 patients of psoriasis and an equal number of age-sex matched controls were included in the study, to rule out any possible role of confounding factors. Most of the patients ( $30 \%$ each) were either from the $31-40$ years or 51-60 years age group. The demographic and clinical data of study subjects has been summarized in the Tab. 1. Eighty percent of patients were suffering with psoriasis vulgaris, the most common variant of psoriasis.

\section{Association of atherogenic risk factors with disease in patients}

The main atherogenic characteristics of patient and control groups are shown in Tab. 2.

\section{Conventional risk factors}

Of the total 80 psoriatic patients studied, $4(5.0 \%)$ had diabetes, 4 (5\%) had hypertension, 54 (60\%) had dyslipidemia and 38 patients (47.5\%) were still actively smoking with mean smoking index (number of cigarettes or beedi/ day $\times$ total duration in years) of $19.28 \pm 8.29$. The mean of the severity of disease (PASI) $(15.60 \pm 10.79)$ in patient group was found to be significantly associated with duration of disease $(\mathrm{p}=0.001)(\mathrm{r}=0.638)$ and waist: hip ratio

Tab. 1: Demographic and clinical evaluation in psoriasis patients and healthy controls.

\begin{tabular}{|c|c|c|c|}
\hline Variables & Control $(n=80)$ & Psoriasis Patients $(n=80)$ & $P$ value \\
\hline \multicolumn{4}{|l|}{ Demographic variables } \\
\hline $\begin{array}{l}\text { Sex (male/female) } \\
\text { Age (years) }\end{array}$ & $\begin{array}{c}60 / 20 \\
41.23 \pm 13.00\end{array}$ & $\begin{array}{c}60 / 20 \\
40.58 \pm 10.58\end{array}$ & $\overline{-}$ \\
\hline \multicolumn{4}{|l|}{ Lifestyle Factors } \\
\hline $\begin{array}{l}\text { Alcohol use (\%) } \\
\text { Smoking (\%) }\end{array}$ & $\begin{array}{l}5 \\
8\end{array}$ & $\begin{array}{l}17.0 \\
47.5\end{array}$ & $\begin{array}{l}<0.050 \\
<0.001\end{array}$ \\
\hline \multicolumn{4}{|c|}{ Anthropometric Variables (mean $\pm \mathrm{SD}$ ) } \\
\hline $\begin{array}{l}\text { Waist/hip ratio } \\
\text { Body Mass Index }\left(\mathrm{kg} / \mathrm{m}^{2}\right)\end{array}$ & $\begin{array}{r}0.82 \pm 0.03 \\
24.18 \pm 3.17\end{array}$ & $\begin{array}{r}0.90 \pm 0.09 \\
24.76 \pm 3.77\end{array}$ & $\begin{array}{r}<0.001 \\
0.453\end{array}$ \\
\hline \multicolumn{4}{|c|}{ Disease specific characteristics (mean \pm SD) } \\
\hline $\begin{array}{l}\text { Age of onset of disease } \\
\text { Duration of Disease (months) } \\
\text { Psoriasis Area Severity Index }\end{array}$ & & $\begin{array}{l}36.40 \pm 11.24 \\
41.03 \pm 30.72 \\
15.60 \pm 10.79\end{array}$ & $\begin{array}{l}- \\
- \\
-\end{array}$ \\
\hline
\end{tabular}

Tab. 2: Atherogenic risk factor and CIMT measurements in psoriasis patients and healthy controls.

\begin{tabular}{|l|c|c|c|}
\hline Variables & Controls $(\mathrm{n}=80)$ & Psoriasis Patients $(\mathrm{n}=80)$ & P value \\
\hline Systolic Blood Pressure $(\mathrm{mmHg})$ & $113 \pm 2.20$ & $120 \pm 1.90$ & 0.047 \\
\hline Diastolic Blood Pressure $(\mathrm{mmHg})$ & $70 \pm 9.80$ & $78 \pm 10.60$ & 0.009 \\
\hline Blood Sugar (Fasting) (mg/dl) & $83.17 \pm 13.66$ & $93.64 \pm 7.240$ & 0.157 \\
\hline Blood Sugar (PP) (mg/dl) & $108.8 \pm 17.60$ & $130.3 \pm 14.54$ & 0.235 \\
\hline Total Cholesterol (mg/dl) & $144.1 \pm 18.09$ & $198.6 \pm 22.44$ & $<0.001$ \\
\hline HDL-C (mg/dl) & $44.78 \pm 6.84$ & $38.18 \pm 8.25$ & 0.254 \\
\hline LDL-C (mg/dl) & $82.75 \pm 22.64$ & $128.6 \pm 20.92$ & $<0.010$ \\
\hline Triglycerides (mg/dl) & $100.6 \pm 28.72$ & $119.8 \pm 25.52$ & $<0.010$ \\
\hline apolipoprotein A-I (g/l) & $1.96 \pm 0.48$ & $1.80 \pm 0.68$ & 0.235 \\
\hline apolipoprotein B (g/l) & $0.79 \pm 0.32$ & $0.97 \pm 0.39$ & 0.025 \\
\hline apoB/apoA-I ratio & $0.417 \pm 0.14$ & $0.62 \pm 0.23$ & $<0.001$ \\
\hline Serum Leptin (ng/ml) & $8.43 \pm 3.64$ & $16.57 \pm 7.68$ & $<0.001$ \\
\hline Mean CIMT (mm) & $0.48 \pm 0.10$ & $0.65 \pm 0.14$ & $<0.001$ \\
\hline
\end{tabular}

HDL-C - High-density lipoprotein-cholesterol; LDL-C - Low-density lipoprotein-cholesterol; mean CIMT - mean Carotid intima-media thickness. 
$(r=0.412, p<0.01)$. Smoking Index was significantly correlated $(\mathrm{r}=0.483),(\mathrm{p}<0.05)$ to severity $(\mathrm{PASI})$ of disease. The data shows that disease gets severe with time and a change in lifestyle may help in curbing the disease.

\section{Nonconventional risk factors}

Serum apoB levels $(\mathrm{P}<0.05)$ and apoB/apoA-I $(\mathrm{P}<0.001)$ ratio in patients were significantly higher as compared to control subjects while the difference in serum apoA-I concentration of two groups were statistically insignificant. Serum leptin levels in patients of psoriasis (16.57 $\pm 7.68)$ was significantly higher $(\mathrm{p}<0.001)$ than those of healthy controls $(8.43 \pm 3.64)$ (Tab. 2$)$. The study shows considerable correlation $(\mathrm{r}=0.603, \mathrm{p}<0.05)$ between higher serum leptin level and raised waist/hip ratio in patients. However no correlation was observed between serum leptin levels and lipid profile.

\section{CIMT measurement and its correlation with atherogenic parameters}

The mean CIMT values of the right and left carotid artery of the patients were found to be significantly higher $(\mathrm{p}<$ $0.001)$ compared with the controls $(0.658 \pm 0.13 \mathrm{~mm}$ vs 0.489 $\pm 0.09 \mathrm{~mm}$ and $0.656 \pm 0.13 \mathrm{~mm}$ vs $0.482 \pm 0.08 \mathrm{~mm}$ respectively). Among patients, 26 (32.5\%) reported an abnormal increase in CIMT i.e. above $0.7 \mathrm{~mm}$. Data in Tab. 3 shows intra group comparison in patients of psoriasis, based on whether or not the CIMT was more than $0.7 \mathrm{~mm}$. There was significant difference between two subgroups (based on CIMT cut off of $0.7 \mathrm{~mm})$ of patients for CIMT ( $<<0.001)$, apoB/ apoA-I ratio $(\mathrm{p}<0.001)$ and serum leptin $(\mathrm{p}<0.05)$ levels.

We analysed the association between CIMT and other variables studied in the patient and in the control group (Tab. 4). All correlations were standardized for age and BMI. Significantly different trends were found for the correlation of CIMT with leptin in patient group than those in the control group.

Data in Fig. 1 suggests that greater CIMT values were significantly associated with age of patient, age of onset of disease $(\mathrm{p}<0.001)$, smoking index, systolic blood pressure, diastolic blood pressure, serum leptin, apoB concentration $(\mathrm{p}<0.05)$ and apoB/apoA-I $(\mathrm{p}<0.001)$ in patient group. However, CIMT was not found to be correlated to either duration, severity of the disease or serum apoA-I concentration in the patients of psoriasis. Even after adjustment for other confounding factors (smoking index and disease duration) no significant association (partial $\mathrm{R}=0.067, \mathrm{p}=0.07$ ) was observed between CIMT and severity of psoriasis (PASI).

Tab. 3: Anthropometric and laboratory values classified by CIMT in psoriasis patients.

\begin{tabular}{|c|c|c|c|}
\hline Variable & CIMT $>0.70 \mathrm{~mm}(\mathrm{~N}=26)$ & CIMT $<0.70 \mathrm{~mm}(\mathrm{~N}=54)$ & $P$ value \\
\hline Age (years) & $50.62 \pm 11.84$ & $36.70 \pm 11.11$ & 0.001 \\
\hline Waist/hip ratio & $0.92 \pm 0.11$ & $0.90 \pm 0.07$ & 0.613 \\
\hline Body Mass Index $\left(\mathrm{kg} / \mathrm{m}^{2}\right)$ & $24.44 \pm 4.46$ & $25.29 \pm 3.69$ & 0.561 \\
\hline Age of onset (years) & $47.46 \pm 11.96$ & $31.93 \pm 11.46$ & $<0.001$ \\
\hline Duration (months) & $46.69 \pm 44.27$ & $39.00 \pm 36.84$ & 0.593 \\
\hline PASI & $17.07 \pm 11.38$ & $14.11 \pm 10.56$ & 0.439 \\
\hline Smoking Index & $13.54 \pm 21.16$ & $7.24 \pm 12.57$ & 0.336 \\
\hline Systolic blood pressure (mmHg) & $125 \pm 1.99$ & $117 \pm 1.12$ & 0.049 \\
\hline Diastolic blood pressure $(\mathrm{mmHg})$ & $82 \pm 9.60$ & $76 \pm 8.18$ & 0.036 \\
\hline Blood Sugar (Fasting) (mg/dl) & $95.51 \pm 8.53$ & $92.70 \pm 6.46$ & 0.257 \\
\hline Blood Sugar (pp) (mg/dl) & $133.4 \pm 16.49$ & $128.8 \pm 13.56$ & 0.239 \\
\hline Total cholesterol (mg/dl) & $199.8 \pm 30.30$ & $192.4 \pm 21.34$ & 0.549 \\
\hline HDL-C (mg/dl) & $39.63 \pm 7.78$ & $41.39 \pm 8.30$ & 0.519 \\
\hline LDL-C (mg/dl) & $128.5 \pm 28.52$ & $128.0 \pm 22.99$ & 0.951 \\
\hline Triglycerides (mg/dl) & $129.9 \pm 35.54$ & $112.0 \pm 20.7$ & 0.111 \\
\hline apolipoprotein B (g/l) & $1.23 \pm 0.37$ & $0.99 \pm 0.35$ & 0.062 \\
\hline apolipoprotein A-I (g/l) & $1.58 \pm 0.69$ & $1.95 \pm 0.65$ & 0.123 \\
\hline apoB/apoA-I ratio & $0.81 \pm 0.19$ & $0.53 \pm 0.19$ & $<0.001$ \\
\hline Leptin (ng/ml) & $20.58 \pm 7.86$ & $14.64 \pm 6.94$ & 0.030 \\
\hline Mean CIMT (mm) & $0.82 \pm 0.09$ & $0.57 \pm 0.05$ & $<0.001$ \\
\hline
\end{tabular}

PASI - Psoriasis Area Severity Index; HDL-C - High-density lipoprotein-cholesterol; LDL-C - Low-density lipoprotein-cholesterol; CIMT - Carotid intima-media thickness. 
Tab. 4: Correlation of Carotid intima media thickness with cardiovascular risk factors in patients of psoriasis and controls.

\begin{tabular}{|l|c|c|c|}
\hline Variables & Controls $(\mathrm{n}=80)$ & Psoriasis Patients $(\mathrm{n}=80)$ & Equality of correlation coefficients P value \\
\hline Systolic blood pressure & 0.150 & 0.397 & 0.125 \\
\hline Diastolic blood pressure & 0.185 & 0.328 & 0.345 \\
\hline Total Cholesterol & 0.019 & 0.108 & 0.582 \\
\hline Triglycerides & 0.007 & 0.385 & 0.017 \\
\hline HDL cholesterol & -0.171 & -0.085 & 0.590 \\
\hline LDL cholesterol & 0.048 & 0.016 & 0.842 \\
\hline apolipoprotein A1 & -0.141 & -0.194 & 0.737 \\
\hline apolipoprotein B & 0.044 & 0.353 & 0.047 \\
\hline apoB/apoA-I ratio & 0.237 & 0.519 & 0.041 \\
\hline Leptin & -0.168 & 0.399 & $<0.001$ \\
\hline
\end{tabular}

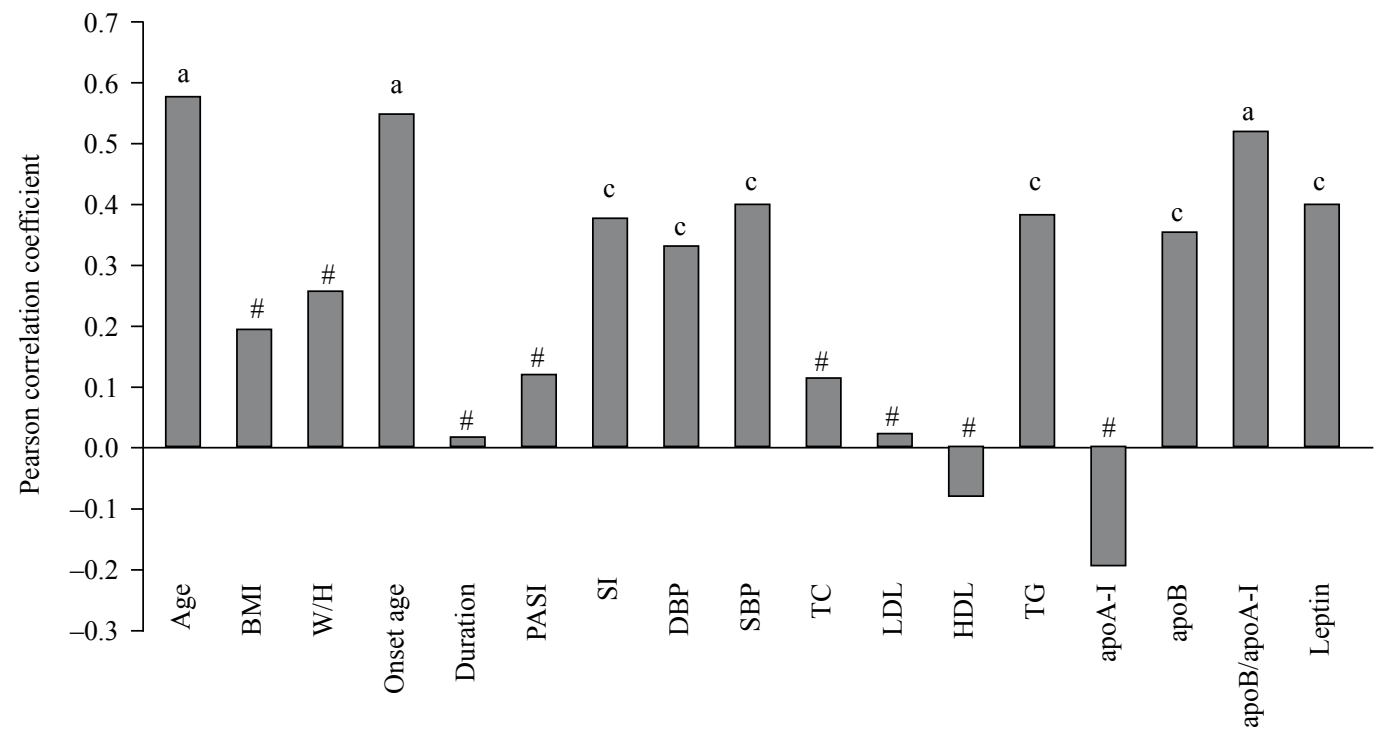

Fig. 1: Bar diagram showing correlation between CIMT and several variables.

BMI - Body Mass Index; W/H - waist/hip ratio; PASI - Psoriasis Area Severity Index; SI - smoking index; DBP - Diastolic blood pressure; SBP - Systolic blood pressure; TC - Total cholesterol; LDL - Low-density lipoprotein; HDL - High-density lipoprotein; TG Triglycerides; apoA-I - apolipoprotein A-I; apoB - apolipoprotein B; apoB/apoA-I - apoB/apoA-I ratio. \# - Non significant; Correlation was significant at the ${ }^{\mathrm{a}} \mathrm{P}<0.001,{ }^{\mathrm{b}} \mathrm{P}<0.01,{ }^{\mathrm{c}} \mathrm{P}<0.05$.

Results from regression analysis $(\mathrm{F}=8.945, \mathrm{P}<0.001)$ suggested that increased CIMT in patients may be associated to cumulative chronic inflammation and impaired apolipoprotein and thus it can serve as a reliable indicator of subclinical disease and may help in early diagnosis of CVD risk factors associated with psoriasis.

The 75th percentile values used to determine high risk values for apoB/apoA-I ratio, leptin levels and mean CIMT were $0.73,23.67 \mathrm{ng} / \mathrm{ml}$ and $0.70 \mathrm{~mm}$ respectively. We calculated predictive values (sensitivity, specificity, positive predictive value (PPV) and negative predictive value (NPV) for each biomarker. The results are as follows: $30 \%, 92.5 \%$, $80 \%$ and $56.92 \%$ for apoB/apoA-I ratio; $25 \%, 90 \%, 71.43 \%$ and $54.55 \%$ for leptin and $30 \%, 92.5 \%, 80 \%$ and $56.92 \%$ for mean CIMT. The predictive values increased $(47.50 \%$,
$82.50 \%, 73.08 \%$ and $61.11 \%$ ) when we took three biomarkers into account (Tab. 5). A fairly significant kappa value of 0.3 depicted that the three biomarkers can be used to assess early risk of cardiovascular disease in psoriasis patients. Assuming 75th percentile value as cut off for leptin, apoB/apoA-I ratio and CIMT, the odds ratio calculated was 4.26 (2.06-8.80 CI). According to results of ROC curves (Fig. 2), the variable with highest AUC (area under curve) was mean CIMT followed by serum leptin and apoB/apoA-I ratio respectively.

\section{Discussion}

Psoriasis and atherosclerosis are systemic inflammatory disease. Psoriasis is not a life-threatening disease by itself 
Tab. 5: Predictive values for biomarkers (Leptin, apoB/apoA-I ratio and CIMT)

\begin{tabular}{|l|c|c|c|}
\hline 95\% CI Lower limit Higher limit \\
\hline Disease prevalence & $50.00 \%$ & $42.00 \%$ & $58.00 \%$ \\
\hline Sensitivity & $47.50 \%$ & $36.21 \%$ & $58.98 \%$ \\
\hline Specificity & $82.50 \%$ & $72.38 \%$ & $90.08 \%$ \\
\hline Positive predictive value & $73.08 \%$ & $58.97 \%$ & $84.42 \%$ \\
\hline Negative predictive value & $61.11 \%$ & $51.25 \%$ & $70.34 \%$ \\
\hline
\end{tabular}

however cardiovascular risk factors associated with the disease, at times, make it fatal $(15,16,17)$. Recently few studies from western countries have reported that psoriasis may be independently associated with myocardial infarction $(18,19)$. Anthropometric and serum lipid measurement has been recommended in psoriasis patients, but their role is not certain till date $(2,19,20,21)$. Thus, we evaluated psoriasis patients for both conventional and non conventional cardiovascular risk factors. Recently, CIMT has been recognised as reliable non invasive diagnostic tool to identify premature atherosclerosis. We studied potential role of apoB/apoA-I ratio and serum leptin levels as predictor for subclinical atherosclerosis, in association with CIMT.

We matched age, sex and BMI in patients and controls to rule out their possible role in evaluation of atherogenic parameters studied.

It was observed that $47.5 \%$ patients were active smoker and nearly $60 \%$ of patients were either overweight (BMI $>25 \mathrm{~kg} / \mathrm{m}^{2}$ ), hypertensive or had abnormal serum lipid profile. Obesity could be sign of inactiveness in these patients. In addition, waist/hip ratio was significantly higher in patients than controls $(\mathrm{p}<0.001)$. In 1995, Henseler and Christophers also reported that patients with severe psoriasis were mostly overweight (22). In a similar study Naldi et al., observed that a moderate increase in BMI was associated with increased risk of psoriasis and clinically obesity doubled the risk of psoriasis (23).

Serum leptin (OB protein) in patient group was significantly related to raised waist hip ratio, PASI score and increased CIMT, thus providing its association with psoriasis. These findings were in agreement with previous results of Enany et al. (24), Cerman et al. (25) and Hamminga et al. (26) who suggested that leptin may serve a role of marker in psoriasis. Thus we may infer that increased serum leptin in obese patients may be correlated to events of metabolic dysfunction.

In the study, apoB levels and apoB/apoA-I ratio were elevated in psoriasis and abnormal apolipoprotein metabolism may be related to the high incidence of atherosclerosis.

Among patients, $32.5 \%$ had high CIMT (above $0.7 \mathrm{~mm}$ ) with the mean CIMT $(0.65 \pm 0.02 \mathrm{~mm})$. Recent study by Kimhi et al. (27) showed that psoriatic arthritis patients had significantly higher CIMT compared with controls $(0.76 \pm$ 0.11 versus $0.64 \pm 0.27, \mathrm{P}<0.00001)$ which was similar

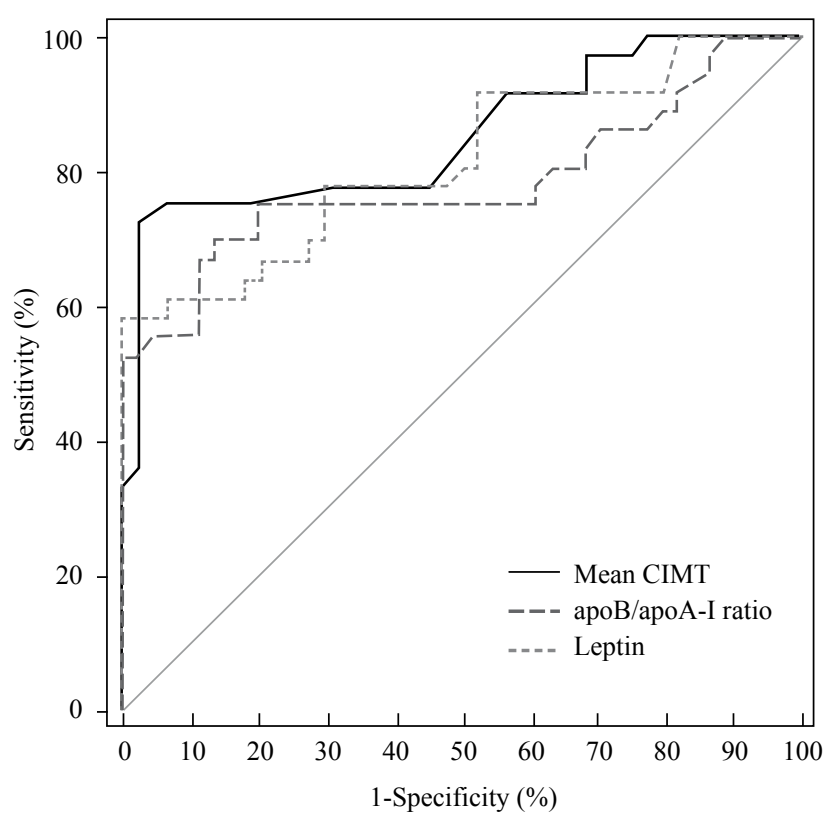

Fig. 2: Comparison of ROC curves among leptin, apoB/apoA-I ratio and CIMT variable.

AUC values were 0.81 (95\% CI, 0.71-0.89); 0.78 (95\% CI, $0.67-$ $0.86)$ and $0.85(95 \% \mathrm{CI}, 0.75-0.92)$ respectively and there was no significant pair wise difference for AUC values $(\mathrm{P}>0.05)$.

to results given by Gonzalez et al. and Mongi et al. (28, 29). In our current study, we have observed a similar trend with significantly higher CIMT $(\mathrm{P}<0.001)$. Grobbee et al. (30) and Tam et al. $(31,32)$ have shown that presence of carotid lesions and thickened intima can be considered as a surrogate marker for the detection of early atherosclerosis in subclinical stage of disease and can be correlated to cardiovascular risks among patients and this increase is significantly associated with clinical (age of onset of the disease) and atherogenic (apoB/apoA-I ratio) parameters as also revealed in our study. We did not find any correlation of CIMT with disease duration or severity which was similar to reports given by Youssef et al. (33). This was perhaps due to quite young age group of our patients and fluctuating course of the disease.

Our finding was concordant to those of Huang et al. who found that both apoB levels and apoB/apoA-I ratio were associated to increased CIMT (34). As reported by Dahlen et al. we also observed that high apoB/apoA-I ratio, and not serum lipid profile, were associated with increased CIMT (35). Previous studies from various countries have also demonstrated that an increased CIMT value often correlates to atherogenic risks in various chronic diseases (36). By regression analysis, we found a significant cumulative effect of apoB/apoA-I ratio and serum leptin (as predictors) on mean value of carotid artery records of psoriasis patients studied (as dependent variables) $(\mathrm{F}=8.945, \mathrm{P}<0.001)$. Thus a significant correlation of cardiovascular risk factors and 
inflammatory biomarkers with increased CIMT in present population may be linked to increased risk of subclinical atherosclerosis in the diseased group. The study further shows that CIMT along with leptin and apoB/apoA-I ratio can be used as biomarkers to estimate cardiac risk in patients of psoriasis.

\section{Conclusions}

We may conclude that the measurement of apoB/apoA-I ratio rather than serum apolipoprotein estimation, can help in diagnosis of premature atherosclerosis in patients of psoriasis. Chronic inflammation plays a major contributing role in both psoriasis and atherosclerosis. This study demonstrates the need to screen patients for serum leptin, apoB/apoA-I ratio and CIMT along with known conventional risk factors, to predict subclinical atherosclerosis and hence the future risk of CVD in patients of psoriasis.

\section{Acknowledgements}

This work was supported by grants from the University Grants Commission, Government of India.

\section{References}

1. Prodanovich S, Kirsner RS, Kravetz JD, Ma F, Martinez L, Federman DG. Association of psoriasis with coronary artery, cerebrovascular, and peripheral vascular disease and mortality. Arch Dermatol 2009; 145: 700-03.

2. Rocha-Pereira P, Santos-Silva A, Rebelo I, et al. Dislipidemia and oxidative stress in mild and in severe psoriasis as a risk for cardiovascular disease. Clin Chim Acta. 2001 Jan; 303(1-2): 33-9.

3. Jellinger PS, Smith DA, Mehta AE, et al. American Association of Clinical Endocrinologists Guidelines for Management of Dyslipidemia and Prevention of Atherosclerosis. Endocr Pract 2012; 18(1): 1-78.

4. Johnston A, Arnadottir S, Gudjonsson JE, et al. Obesity in psoriasis: Leptin and resistin as mediators of cutaneous inflammation. Br J Dermatol 2008; 159: 342-50.

5. Fujita Y, Murakami M, Ogawa Y, et al. Leptin inhibits stress-induced apoptosis of T lymphocytes. Clin Exp Immunol 2002; 128: 21-26.

6. Aktan F, Rota S, Erdogan BS, et al. A Role of leptin in psoriasis? Turk J Med Sci 2007; 37(3): 135-38.

7. Asanuma Y, Oeser A, Shintani AK, et al. Premature coronary-artery atherosclerosis in systemic lupus erythematosus. N Engl J Med 2003; 349: 2407-15.

8. Belibou C, Ancuța C, Ancuţa E, Filos C, Chirieac R. Carotid intima-media thickness and plaque as surrogate biomarkers of atherosclerosis among consecutive women with systemic lupus erythematosus. Rom J Morphol Embryol 2012; 53 : 29-34.

9. Expert Panel on Detection, Evaluation and Treatment of High Blood Cholesterol in Adults. Executive summary of the third report of the national cholesterol education program (NCEP) expert panel on detection, evaluation, and treatment of high blood cholesterol in adults (Adult Treatment Panel III). JAMA 2001; 285: 2486-97.

10. Einhorn D, Reaven GM, Cobin RH, et al. American College of Endocrinology position statement on the insulin resistance syndrome. Endocr Pract 2003; 9: 237-52.

11. Alberti KG, Zimmet P, Shaw J. IDF. Epidemiology Task Force Consensus Group The metabolic syndrome - a new worldwide definition. Lancet 2005; 366: 1059-62.
12. Fredriksson T, Pettersson U. Severe psoriasis-oral therapy with a new retinoid. Dermatologica 1978; 157: 238-44.

13. Votila M, Ruoslanti E, Enguall E. Immunology methods. J Immunol Methods 1981; 42: 11-15.

14. Huang Y, Bi Y, Wang W, et al. Glycated hemoglobin A1c, fasting plasma glucose, and two-hour postchallenge plasma glucose levels in relation to carotid intima-media thickness in chinese with normal glucose tolerance. J Clin Endocrinol Metab 2011; 96: 1461-65.

15. Mok CC, Ko GT, Ho LY, Yu KL, Chan PT, To CH. Prevalence of atherosclerotic risk factors and the metabolic syndrome in patients with chronic inflammatory arthritis. Arthritis Care Res 2011; 63: 195-202.

16. Cohen AD, Sherf M, Vidavsky L, Vardy DA, Shapiro J, Meyerovitch J. Association between psoriasis and the metabolic syndrome. A cross-sectional study. Dermatol 2008; 216: 152-55.

17. Neimann AL, Shin DB, Wang X, Margolis DJ, Troxel AB, Gelfand JM. Prevalence of cardiovascular risk factors in patients with psoriasis. J Am Acad Dermatol 2006; 55: 829-35.

18. Shapiro J, Cohen AD, David M, et al. The association between psoriasis, diabetes mellitus, and atherosclerosis in Israel: a case-control study. J Am Acad Dermatol 2007; 56: 629-34.

19. Gelfand JM, Neimann AL, Shin DB, Wang X, Margolis DJ, Troxel AB. Risk of myocardial infarction in patients with psoriasis. J Am Med Assoc 2006; 296: 1735-41.

20. Seishima M, Seishima M, Mori S, Noma A. Serum lipid and apolipoprotein levels in patients with psoriasis. Br J Dermatol 1994; 130: 738-42.

21. Bajaj DR, Mahesar SM, Devrajani BR, Iqbal MP. Lipid profile in patients with psoriasis presenting at Liaquat University Hospital Hyderabad. J Pak Med Assoc 2009; 59(8): 512-15.

22. Henseler T, Christophers E. Disease concomitance in psoriasis. J Am Acad Dermatol 1995; 32: 982-86.

23. Naldi L, Chatenoud L, Linder D, et al. Cigarette smoking, body mass index, and stressful life events as risk factors for psoriasis: results from an Italian case-control study. J Invest Dermatol 2005; 125: 61-67.

24. Enany B, El Zohiery AK, Elhilaly R, Badr T. Carotid intima-media thickness and serum leptin in psoriasis. Herz 2012; 37: 527-33.

25. Cerman AA, Bozkurt S, Sav A, Tulunay A, Elbasi MO, Ergun T. Serum leptin levels, skin leptin and leptin receptor expression in psoriasis. Br J Dermatol 2008; 159: 820-26.

26. Hamminga EA, Lely AJ, Neumann HA, Thio HB. Chronic inflammation in psoriasis and obesity: implication for therapy. Med Hypotheses 2006; 67: 768-73.

27. Kimhi O, Caspi D, Bornstein NM, et al. Prevalence and risk factors of atherosclerosis in patients with psoriatic arthritis. Semin Arthritis Rheum 2007; 36: 203-09.

28. Gonzalez JC, Llorca J, Amigo DE, Dierssen T, Martin J, Gonzalez GMA. High prevalence of subclinical atherosclerosis in psoriatic arthritis patients without clinically evident cardiovascular disease or classic atherosclerosis risk factors. Arthritis Rheum 2007; 57: 1074-80.

29. El-Mongy S, Fathy H, Abdelaziz A, et al. Subclinical atherosclerosis in patients with chronic psoriasis: a potential association. J Eur Acad Dermatol Venereol 2010; 24: 661-66.

30. Grobbee DE, Bots ML. Carotid artery intima-media thickness as an indicator of generalized atherosclerosis. J Intern Med 1994; 236: 567-73.

31. Tam LS, Shang Q, Li EK, et al. Subclinical carotid atherosclerosis in patients with psoriatic arthritis. Arthritis Rheum 2008; 59: 1322-31.

32. Tam LS, Tomlinson B, Chu TT, et al. Cardiovascular risk profile of patients with psoriatic arthritis compared to controls-the role of inflammation. Rheumatol 2008; 47: 718-23.

33. Youssef SS, Hosny S, Bakr I, Youssef A. Susceptibility to atherosclerosis in patients with psoriasis and psoriatic arthritis as determined by increased carotid artery intima-media thickness. J Pan-Arab League of Dermatologists 2009; 20: 159-72.

34. Huang F, Yang Z, Xu B, et al. Both serum ApoB and the ApoB/apoA-I ratio are associated with carotid intima-media thickness. PloS ONE 2013; 8(1): e54628.

35. Dahlen EM, Lanne T, Engvall J, et al. Carotid intima-media thickness and apoB apoA-I ratio in middle-aged patients with Type 2 diabetes. Diabet Med 2009; 26 : 384-90.

36. Doria A, Shoenfeld Y, Wu R, et al. Risk factors for subclinical atherosclerosis in a prospective cohort of patients with systemic lupus erythematosus. Ann Rheum Dis 2003; 62: 1071-77.

Suman Bala Sharma, Department of Biochemistry, University College of Medical Sciences (University of Delhi) and G. T. B. Hospital, Dilshad Garden, Delhi, India; e-mail: drsbs08@hotmail.com 